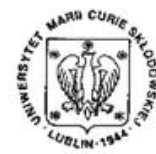

Annales UMCS Informatica AI XIII, 1 (2013) 37-51 DOI: $10.2478 / \mathrm{v} 10065-012-0045-8$

Annales UMCS

Informatica

Lublin-Polonia

Sectio AI

http://www.annales.umcs.lublin.pl/

\title{
Exact difference schemes and schemes of higher order of approximation for convection-diffusion equation. I
}

\author{
Magdalena Lapinska-Chrzczonowicz ${ }^{1 *}$, Piotr Matus $^{1,2}$ \\ ${ }^{1}$ Institute of Mathematics and Computer Science, \\ John Paul II Catholic University of Lublin, \\ Al. Ractawickie 14, 20-950 Lublin, Poland \\ ${ }^{2}$ Institute of Mathematics NAS of Belarus, \\ 11 Surganov Str., 220072 Minsk, Belarus
}

\begin{abstract}
The initial-boundary value problem for a convection-diffusion equation

$$
\begin{gathered}
\frac{\partial u}{\partial t}+a \frac{\partial u}{\partial x}=\frac{\partial}{\partial x}\left(k(u) \frac{\partial u}{\partial x}\right),(x, t) \in Q_{T}, \\
u(x, 0)=u_{0}(x), 0 \leq x \leq l, u(0, t)=\mu_{1}(t), u(l, t)=\mu_{2}(t), 0 \leq t \leq T
\end{gathered}
$$
\end{abstract}

is considered. The difference scheme, approximating this problem, is constructed. It is shown that for traveling wave solutions the scheme is exact (EDS). The monotonicity of the scheme is also taken into consideration. Presented numerical experiments illustrate the theoretical results investigated in the paper.

\section{Introduction}

In constructing a difference scheme the main aim is to approximate an original problem with a prescribed accuracy in a finite number of operations. In this regard, the question of the approximation order of the difference scheme arises at once. The order of the approximation is desired to be as high as possible with a minimum number of grid nodes in the pattern of a scheme at the same time. In some cases, the EDS can be constructed.

Definition 1. A difference scheme is exact if the truncation error equals zero or $y=u$ at the grid nodes.

*lapkam@kul.pl 
Let us introduce the uniform grids $\bar{\omega}_{h}=\left\{x_{i}=i h, i=0, \ldots, N, h N=l\right\}, \bar{\omega}_{\tau}=$ $\omega_{\tau} \cup\{0\}=\left\{t_{n}=n \tau, n=0, \ldots, N_{0}, \tau N_{0}=T\right\}, \bar{\omega}_{h \tau}=\bar{\omega}_{h} \times \bar{\omega}_{\tau}$. Here and after $\omega_{h \tau}=$ $Q_{T} \cap \bar{\omega}_{h \tau}$. In the paper we will use the following notation [1]

$$
\begin{gathered}
\hat{y}=y_{i}^{n+1}, y=y_{i}^{n}, \bar{y}=y_{i}^{n+1 / 2}, y^{(\sigma)}=\sigma y_{i}^{n+1}+(1-\sigma) y_{i}^{n}, y_{t}=\left(y_{i}^{n+1}-y_{i}^{n}\right) / \tau, \\
y_{x}=\left(y_{i+1}-y_{i}\right) / h, y_{\bar{x}}=\left(y_{i}-y_{i-1}\right) / h, y_{\bar{x} x}=\left(y_{i+1}-2 y_{i}+y_{i-1}\right) / h^{2} .
\end{gathered}
$$

Let $C_{n}^{m}\left(\bar{Q}_{T}\right)$ be the class of functions with $m$ derivatives in $x$ and $n$ derivatives in $t$, all necessary derivatives being continuous in the domain $\bar{Q}_{T}=Q_{T} \cup \partial Q_{T}$.

Contracting EDSs was, for example, discussed in $[2, \mathbf{3}, \mathbf{4}, \mathbf{5}, \mathbf{6}]$. In the papers by R.E. Mickens certain rules for construction of the nonstandard finite difference schemes are given. In [2] there were constructed EDSs from which the implementable truncated difference schemes were derived. For the Cauchy problem

$$
\frac{d u}{d t}=f_{1}(t) f_{2}(u), u=u(t), 0<t \leq T, u(0)=u_{0},
$$

the following EDS can be constructed [7]

$$
\frac{y^{n+1}-y^{n}}{\tau}=\frac{1}{\tau} \int_{t_{n}}^{t_{n+1}} f_{1}(t) d t\left(\frac{1}{y^{n+1}-y^{n}} \int_{y^{n}}^{y^{n+1}} \frac{d u}{f(u)}\right)^{-1}, t \in \omega_{\tau}, y^{0}=u_{0} .
$$

In the above scheme the special Steklov averaging is used.

The authors have established that for the parabolic problems with travelling wave solutions the EDS may be constructed $[\mathbf{7}, \mathbf{8}]$. This paper refers to the travelling waves which arise in many problems such as heat transfer, combustion, reaction chemistry, fluid dynamics, plasma physics, soil-moisture, foam drainage, crystal growth, biological population genetics, cellular ecology, neurology and synergy $[\mathbf{9}, \mathbf{1 0}]$. This was the motivation for constructing EDS for a convection-diffusion problem. The authors took advantage of the equivalence of the convection-diffusion equation to the transport equation for travelling wave solutions and constructed the difference scheme which can be reduced to the EDS for transport problem in this special case. The main goal was to construct the difference scheme which can be applied not only for a class of travelling wave solutions but also for a wide class of solutions. The monotonicity of the scheme was also an important question. For further investigation the corollary of the maximum principle was used, which asserts that for a solution to the difference equation

$$
\begin{gathered}
A_{i} y_{i-1}-C_{i} y_{i}+B_{i} y_{i+1}=-F_{i}, i=1, \ldots, N-1, \\
y_{0}=\mu_{1}, y_{N}=\mu_{2},\left|A_{i}\right| \neq 0,\left|B_{i}\right| \neq 0,
\end{gathered}
$$

the estimate

$$
\|y\|_{\bar{C}} \leq \max \left\{\left|\mu_{1}\right|,\left|\mu_{2}\right|,\|F / D\|_{C}\right\}
$$


is valid if $D_{i}=\left|C_{i}\right|-\left|A_{i}\right|-\left|B_{i}\right|>0$ [11]. The equation (1) can be rewritten in a more general unified canonical form [11]

$$
A(P) y(P)=\sum_{Q \in P^{\prime} t t^{\prime}(P)} B(P, Q) y(Q)+F(P), P \in \omega,
$$

where $A(P), B(P, Q), F(P)$ are given grid functions, $\operatorname{Patt}^{\prime}(P)=\operatorname{Patt}(P) \backslash\{P\}$, and Patt is the difference pattern.

Definition 2. [11] The difference scheme (3) is monotone if $A(P)>0, B(P, Q)>0$ for all $P \in \omega, Q \in \operatorname{Patt}^{\prime}(P)$ and

$$
D(P)=A(P)-\sum_{Q \in \operatorname{Patt}^{\prime}(P)} B(P, Q) \geq 0 .
$$

The outline of this paper is as follows. In the first two sections the EDS for transport problems are considered. On their basis the EDS for a convection-diffusion problem is constructed in the next section and the approximation order is considered. The numerical results are given and the future research directions are discussed.

\section{Difference schemes for a homogenous transport equation}

In the domain $\bar{Q}_{T}=\{(x, t): x \in[0, l], t \in[0, T]\}$ let us consider the initialboundary value problem for a transport equation with a positive coefficient $a>0$

$$
\begin{gathered}
\frac{\partial u}{\partial t}+a \frac{\partial u}{\partial x}=0,(x, t) \in Q_{T}, \\
u(x, 0)=u_{0}(x), 0 \leq x \leq l, \quad u(0, t)=\mu_{1}(t), u(l, t)=\mu_{2}(t), 0<t \leq T,
\end{gathered}
$$

where $Q_{T}=\{(x, t): x \in(0, l], t \in(0, T]\}$. It is well-known that the solution of the problem (4) is a travelling wave

$$
u(x, t)=U(x-a t),
$$

where $a$ is the wave velocity and $U(\xi)$ is the differentiable function [11].

In this section we review some of the difference schemes approximating the problem (4) - (5) with particular reference to an exactness property. The difference schemes introduced below are exact under the same condition connected with the Courant number $\gamma$

$$
\gamma=a \tau / h=1
$$

Let us begin with the well-studied explicit difference scheme $[\mathbf{1 1}, \mathbf{1 2}]$

$$
\begin{gathered}
y_{t}+a y_{\bar{x}}=0, \\
y_{i}^{0}=u_{0}\left(x_{i}\right), x_{i} \in \bar{\omega}_{h}, \quad y_{0}^{n+1}=\mu_{1}\left(t_{n+1}\right), y_{N}^{n+1}=\mu_{2}\left(t_{n+1}\right), t_{n+1} \in \omega_{\tau} .
\end{gathered}
$$

This scheme is monotone and stable for $\gamma \leq 1$. Another scheme $[\mathbf{1 1}, \mathbf{1 2}]$

$$
y_{t}+a \hat{y}_{x}=0
$$


is monotone and stable with a contrary condition ie. $\gamma=a \tau / h \geq 1$. The above schemes have the first order of approximation $\psi_{i}^{n}=O(\tau+h)$ for $\gamma \neq 1$. The third scheme approximating problem (4) - (5) is weighted simultaneously with respect to the time and space and has the form

$$
y_{(\alpha) t}+a y_{\bar{x}}^{(\sigma)}=0, \alpha, \sigma \in[0,1] .
$$

Here $y_{(\alpha)}=\alpha y_{i}^{n}+(1-\alpha) y_{i-1}^{n}$. This scheme is also exact under the condition $\gamma=1$, but only for a special case of weights, ie. $\alpha+\sigma=1$. It has the second order of approximation $O\left(\tau^{2}+h^{2}\right)$ for $\alpha=\sigma=0.5$ and $\gamma \neq 1$. Furthermore, it is monotone and stable under the condition $\alpha+\gamma \sigma \geq \max \{\gamma, 1\}$ [13]. Now we deal with another difference scheme weighted simultaneously with respect to the time and space

$$
y_{t}+a \sigma \hat{y}_{x}+a(1-\sigma) y_{\bar{x}}=0 .
$$

This scheme is a combination of the schemes (6) and (8). It is monotone and stable for $[8]$

$\sigma=\sigma_{i}(\gamma)= \begin{cases}0, & \text { if } \gamma \leq 1, i=1, \ldots, N, \\ 1, & \text { if } \gamma \geq 1, i=0, \\ \sigma_{1}, & \text { if } \sigma_{\gamma} \leq \sigma_{1}<1, \gamma>1, i=1, \ldots, N-1, \sigma_{\gamma}=\max \{1 / \gamma, 1-1 / \gamma\} .\end{cases}$

This scheme is particularly important in view of next sections. Because of it, we will recall the following lemmas.

Lemma 1. If $\gamma=1$, then the difference scheme (10), (7) is exact.

Proof. Multiplying equations (8) and (6) by $\sigma$ and $1-\sigma$ respectively, and adding the obtained formulas, we get the equation (10). Thus scheme (10), (7) is also exact for $\gamma=1$.

Lemma 2. The approximation error of the difference scheme (10), (7) is

$$
\psi_{i}^{n}= \begin{cases}0, & \text { if } \gamma=1, \\ O\left(\tau^{2}+h^{2}\right), & \text { if } \sigma=0.5, i=0, \ldots, N-1, \gamma \neq 1, \\ O(\tau+h), & \text { if } \sigma \neq 0.5, i=0, \ldots, N-1, \gamma \neq 1\end{cases}
$$

Proof. Lemma 1 shows that $\psi_{i}^{n}=0$ for $\gamma=1$. Now, let $\gamma \neq 1$. The approximation error fulfills the relation

$$
\begin{gathered}
\psi_{i}^{n}=-u_{t, i}+\sigma \frac{\partial \hat{u}}{\partial t}+(1-\sigma) \frac{\partial u}{\partial t}-a \sigma \hat{u}_{x, i}+a \sigma \frac{\partial \hat{u}}{\partial x}-a(1-\sigma) u_{\bar{x}, i}+a(1-\sigma) \frac{\partial u}{\partial x}= \\
=\psi_{1 i}^{n}+\psi_{2 i}^{n}+\psi_{3 i}^{n},
\end{gathered}
$$

where

$$
\begin{gathered}
\psi_{1 i}^{n}=-u_{t, i}+\sigma \frac{\partial \hat{u}}{\partial t}+(1-\sigma) \frac{\partial u}{\partial t}, \\
\psi_{2 i}^{n}=-a \sigma \hat{u}_{x, i}+a \sigma \frac{\partial \hat{u}}{\partial x}, \quad \psi_{3 i}^{n}=-a(1-\sigma) u_{\bar{x}, i}+a(1-\sigma) \frac{\partial u}{\partial x} .
\end{gathered}
$$


Our first goal is to estimate the error $\psi_{1 i}^{n}$. The difference operator $u_{t}$ has a Taylor expansion of the form $u_{t, i}=\frac{\partial \bar{u}}{\partial t}+O\left(\tau^{2}\right)$, so the approximation error $\psi_{1 i}^{n}$ is

$$
\psi_{1 i}^{n}=-\frac{\partial \bar{u}}{\partial t}+\sigma \frac{\partial \hat{u}}{\partial t}+(1-\sigma) \frac{\partial u}{\partial t}+O\left(\tau^{2}\right) .
$$

Next, let us estimate the error $\psi_{2 i}^{n}$. In view of the Taylor expansion $\hat{u}_{x, i}=\frac{\partial \hat{u}}{\partial x}+\frac{h}{2} \frac{\partial^{2} \hat{u}}{\partial x^{2}}+$ $O\left(h^{2}\right)$, we have

$$
\psi_{2 i}^{n}=-a \sigma \frac{h}{2} \frac{\partial^{2} \hat{u}}{\partial x^{2}}+O\left(h^{2}\right) .
$$

Analogously, we can show that

$$
\psi_{3 i}^{n}=a(1-\sigma) \frac{h}{2} \frac{\partial^{2} u}{\partial x^{2}}+O\left(h^{2}\right) .
$$

The trick of the proof is to estimate the expression $\psi_{2 i}^{n}+\psi_{3 i}^{n}$. The key relation is

$$
v\left(t_{n+1}\right)=v\left(t_{n}\right)+\tau v^{\prime}\left(t_{n}+\theta_{n}\left(t_{n+1}-t_{n}\right)\right), 0<\theta_{n}<1, \theta_{n}=\text { const. }
$$

The only, but crucial use of the above equality is that from (11) - (13) we obtain the estimations

$$
\begin{gathered}
\psi_{1 i}^{n}=(\sigma-0.5) \tau \frac{\partial^{2} \bar{u}}{\partial t^{2}}+O\left(\tau^{2}\right), \\
\psi_{2 i}^{n}+\psi_{3 i}^{n}=-a(\sigma-0.5) h \frac{\partial^{2} \bar{u}}{\partial x^{2}}+O\left(\tau^{2}+h^{2}\right) .
\end{gathered}
$$

It is straightforward to show that the error of approximation is

$$
\psi_{i}^{n}=O\left((\sigma-0.5)(\tau+h)+\tau^{2}+h^{2}\right) .
$$

Thus, the difference scheme (10), (7) has the second order of approximation for $\sigma=$ 0.5 .

\section{Difference schemes for a semilinear transport equation}

In the domain $\bar{Q}_{T}$ consider a semilinear transport equation

$$
\frac{\partial u}{\partial t}+a \frac{\partial u}{\partial x}=f_{1}(x, t) f_{2}(u), f_{2}(u) \neq 0,(x, t) \in Q_{T},
$$

with the initial and boundary conditions (5). The difference scheme approximating the above problem is [8]

$$
\begin{gathered}
y_{t, i}+a \sigma y_{x, i}^{n+1}+a(1-\sigma) y_{\bar{x}, i}^{n}=\sigma \varphi_{i+1}^{n}+(1-\sigma) \varphi_{i}^{n}, \\
y_{i}^{0}=u_{0}\left(x_{i}\right), x_{i} \in \bar{\omega}_{h}, \quad y_{0}^{n+1}=\mu_{1}\left(t_{n+1}\right), y_{N}^{n+1}=\mu_{2}\left(t_{n+1}\right), t_{n+1} \in \omega_{\tau},
\end{gathered}
$$

where

$$
\varphi_{i}^{n}=\frac{1}{\tau} \int_{t_{n}}^{t_{n+1}} f_{1}\left(x_{i}(t), t\right) d t\left(\frac{1}{y_{i}^{n+1}-y_{i-1}^{n}} \int_{y_{i-1}^{n}}^{y_{i}^{n+1}} \frac{d u}{f(u)}\right)^{-1}, x_{i}(t)=a t+x_{i}(0) .
$$

It is exact for $\gamma=1[7,8]$. 
If the integrals in the EDS cannot be evaluated exactly, to approximate them the Euler-Maclaurin formula can be applied [14]. In this case, instead of EDS, we obtain a difference scheme of an arbitrary order of accuracy.

Example. We consider the function $u(x, t)=\sin ^{2}(\pi(x-t)) /\left(1-t \sin ^{2}(\pi(x-t))\right)$ (see Fig. 1) that satisfies the problem (17), (5) in the domain $\bar{Q}_{T}=[0,1] \times[0,0.9]$ with $a=1, f_{1} \equiv 1$ and $f_{2}(u)=u^{2}[\mathbf{1 5}]$. The corresponding difference scheme is

$$
y_{t, i}+\sigma y_{x, i}^{n+1}+(1-\sigma) y_{\bar{x}, i}^{n}=\sigma y_{i+1}^{n+1} y_{i}^{n}+(1-\sigma) y_{i}^{n+1} y_{i-1}^{n} .
$$

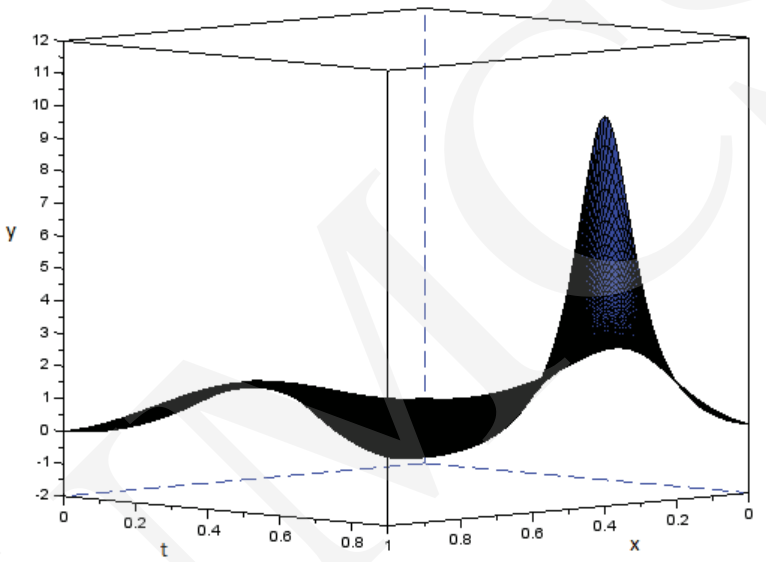

Fig. 1. The approximate solution for $\sigma=0.5, \tau=h=0.01$.

Table 1 presents the numerical results for time and space steps satisfying the condition $\gamma=1$ and demonstrates the exactness of the difference scheme.

Table 1. Numerical results for $\sigma=0.5$ and $\gamma=1$.

\begin{tabular}{|l|l|c|}
\hline$h$ & $\tau$ & $\max _{t_{n} \in \bar{\omega}_{\tau}}\left\|y^{n}-u^{n}\right\|_{\bar{C}}$ \\
\hline 0.1 & 0.1 & $6.94 \cdot 10^{-18}$ \\
\hline 0.01 & 0.01 & $2.78 \cdot 10^{-17}$ \\
\hline
\end{tabular}

Table 2 presents the numerical results for the different time and space steps for which $\gamma \leq 1$ and demonstrates the second order convergence of the difference scheme.

\section{Exact difference scheme for a convection-diffusion in a one-dimensional problem}

In this section the convection-diffusion equation is considered and the difference scheme is constructed. This scheme can be applied for a wide class of solutions but 
Table 2. Numerical results for $\sigma=0.5$ and $\gamma \neq 1$.

\begin{tabular}{|l|l|c|}
\hline$h$ & $\tau$ & $\max _{t_{n} \in \bar{\omega}_{\tau}}\left\|y^{n}-u^{n}\right\|_{\bar{C}}$ \\
\hline 0.05 & 0.09 & $2.33 \cdot 10^{+00}$ \\
\hline 0.01 & 0.018 & $1.50 \cdot 10^{-01}$ \\
\hline 0.005 & 0.009 & $3.82 \cdot 10^{-02}$ \\
\hline 0.001 & 0.0018 & $1.54 \cdot 10^{-03}$ \\
\hline
\end{tabular}

it is particularly important for travelling wave solutions. In this case the constructed scheme is exact.

In the domain $\bar{Q}_{T}$ let us consider the boundary-value problem for the convectiondiffusion equation

$$
\begin{gathered}
\frac{\partial u}{\partial t}+a \frac{\partial u}{\partial x}=\frac{\partial}{\partial x}\left(k(u) \frac{\partial u}{\partial x}\right),(x, t) \in Q_{T}, a>0, \\
u(x, 0)=u_{0}(x), 0 \leq x \leq l, \\
u(0, t)=\mu_{1}(t), u(l, t)=\mu_{2}(t), 0 \leq t \leq T,
\end{gathered}
$$

where $0<c_{1} \leq k(u) \leq c_{2}, c_{1}, c_{2}=$ const, for $u$ in the range of values of the exact solution or its small neighborhood, i.e., $u \in \bar{D}_{\tilde{u}}$. Let us assume that the problem (20) - (22) has a unique solution $u \in C_{3}^{4}\left(\bar{Q}_{T}\right)$ satisfying the condition $0<m_{1} \leq$ $u(x, t) \leq m_{2},(x, t) \in \bar{Q}_{T}, m_{1}, m_{2}=$ const, and all necessary derivatives exist and are continuous and bounded. Moreover let us assume that $k(u) / u \in C^{3}\left(\bar{D}_{\tilde{u}}\right),(x, t) \in Q_{T}$ has continuous and bounded derivatives. Here $Q_{T}=\{(x, t): x \in(0, l), t \in(0, T]\}$. Let us notice that the equation (20) can be rewritten in the equivalent form

$$
\frac{\partial u}{\partial t}+a \frac{\partial u}{\partial x}=\frac{\partial}{\partial x}\left(u \frac{\partial \varphi(u)}{\partial x}\right), \quad \varphi(u)=\int_{u_{0}}^{u} \frac{k(\xi)}{\xi} d \xi
$$

On the uniform grid $\bar{\omega}_{h \tau}$ the problem (23), (21), (22) is approximated by the difference scheme with the weight

$$
\begin{gathered}
y_{t, i}+a y_{\bar{x}, i}^{n}=\sigma \Lambda y_{i}^{n+1}+(1-\sigma) \Lambda^{-} y_{i}^{n},\left(x_{i}, t_{n}\right) \in \omega_{h \tau}, \\
y_{i}^{0}=u_{0}\left(x_{i}\right), x_{i} \in \bar{\omega}_{h}, \\
y_{0}^{n+1}=\mu_{1}\left(t_{n+1}\right), y_{N}^{n+1}=\mu_{2}\left(t_{n+1}\right), t_{n+1} \in \omega_{\tau},
\end{gathered}
$$

where the operators $\Lambda, \Lambda^{-}$are given by the formulas

$$
\Lambda y_{i}=\left(y[\varphi(y)]_{\bar{x}}\right)_{x, i}, \Lambda^{-} y_{i}=\left(y[\varphi(y)]_{x}\right)_{\bar{x}, i}, i=\overline{1, N-1} .
$$

It is worth to noticing here that the explicit scheme $(\sigma=0)$ is also exact but unstable. The experiments show that, when there is no restriction on the grid steps, the weighted scheme (24) - (26) is unstable for $\sigma<0.5$. 
Let us denote the error of the method by $z=y-u$. Then the difference problem for this error takes the following form

$$
\begin{gathered}
z_{t, i}+a z_{\bar{x}, i}^{n}-\sigma \Lambda z_{i}^{n+1}-(1-\sigma) \Lambda^{-} z_{i}^{n}- \\
-\sigma\left(z^{n+1}\left(u^{n+1} \varphi^{\prime}\left(z_{\theta 1}^{n+1}\right)\right)_{\bar{x}}\right)_{x, i}-\sigma\left(u^{n+1}\left(z^{n+1} \varphi^{\prime}\left(u_{\theta 2}^{n+1}\right)\right)_{\bar{x}}\right)_{x, i}- \\
-(1-\sigma)\left(z^{n}\left(u^{n} \varphi^{\prime}\left(z_{\theta 3}^{n}\right)\right)_{x}\right)_{\bar{x}, i}-(1-\sigma)\left(u^{n}\left(z^{n} \varphi^{\prime}\left(u_{\theta 4}^{n}\right)\right)_{x}\right)_{\bar{x}, i}=\psi_{i}^{n}, \quad\left(x_{i}, t_{n}\right) \in \omega_{h \tau}, \\
z_{i}^{0}=0, x_{i} \in \bar{\omega}_{h}, z_{0}^{n+1}=z_{N}^{n+1}=0, t_{n+1} \in \omega_{\tau},
\end{gathered}
$$

where $z_{\theta 1, i}^{n+1}=z_{i}^{n+1}+\theta_{1 i}^{n+1} u_{i}^{n+1}, z_{\theta 3, i}^{n}=z_{i}^{n}+\theta_{3 i}^{n} u_{i}^{n}, u_{\theta 2, i}^{n+1}=u_{i}^{n+1}+\theta_{2 i}^{n+1} z_{i}^{n+1}, u_{\theta 4, i}^{n}=$ $u_{i}^{n}+\theta_{4 i}^{n} z_{i}^{n}$ and the approximation error is

$$
\psi_{i}^{n}=-u_{t, i}-a u_{\bar{x}, i}^{n}+\sigma \Lambda u_{i}^{n+1}+(1-\sigma) \Lambda^{-} u_{i}^{n} .
$$

Here and after $\theta_{p i}^{n}=$ const, $0<\theta_{p i}^{n}<1$.

The considered scheme is monotone and stable in a linear approximation for $\tau \leq$ $h^{2} /(a h+2 b(1-\sigma))$ and $0 \leq \sigma \leq 1$. Moreover, it is also stable for $\sigma \geq 1$ and $a \sigma \tau / h \leq 1$. In this case the linear approximation is treated as a finite difference scheme $y_{t}+a y_{\bar{x}}=b y_{\bar{x} x}^{(\sigma)}$, where $a, b$ are the positive constants.

\subsection{Approximation of the scheme (24) - (26)}

First we prove the theorem on an order of approximation of the difference scheme (24) - (26).

Theorem 1. The approximation error of the difference scheme $(24)-(26)$ is $O(h+$ $\tau)$.

PROOF. The approximation error of the difference scheme (24) - (26) satisfies the relation

$$
\begin{gathered}
\psi_{i}^{n}=-u_{t, i}+\sigma \frac{\partial \hat{u}}{\partial t}+(1-\sigma) \frac{\partial u}{\partial t}-a u_{\bar{x}, i}+a(1-\sigma) \frac{\partial u}{\partial x}+a \sigma \frac{\partial \hat{u}}{\partial x}+ \\
+\sigma \Lambda \hat{u}_{i}-\sigma \frac{\partial}{\partial x}\left(\hat{u} \frac{\partial \varphi(\hat{u})}{\partial x}\right)+(1-\sigma) \Lambda^{-} u_{i}-(1-\sigma) \frac{\partial}{\partial x}\left(u \frac{\partial \varphi(u)}{\partial x}\right)= \\
=\psi_{1 i}^{n}+\psi_{2 i}^{n}+\psi_{3 i}^{n}+\psi_{4 i}^{n},
\end{gathered}
$$

where

$$
\begin{gathered}
\psi_{1 i}^{n}=-u_{t, i}+\sigma \frac{\partial \hat{u}}{\partial t}+(1-\sigma) \frac{\partial u}{\partial t}, \quad \psi_{2 i}^{n}=-a u_{\bar{x}, i}+a \sigma \frac{\partial \hat{u}}{\partial x}+a(1-\sigma) \frac{\partial u}{\partial x} \\
\psi_{3 i}^{n}=\sigma \Lambda \hat{u}_{i}-\sigma \frac{\partial}{\partial x}\left(\hat{u} \frac{\partial \varphi(\hat{u})}{\partial x}\right), \quad \psi_{4 i}^{n}=(1-\sigma) \Lambda^{-} u_{i}-(1-\sigma) \frac{\partial}{\partial x}\left(u \frac{\partial \varphi(u)}{\partial x}\right) .
\end{gathered}
$$

It was shown in Lemma 2 that

$$
\psi_{1 i}^{n}=(\sigma-0.5) \tau \frac{\partial^{2} \bar{u}}{\partial t^{2}}+O\left(\tau^{2}\right) .
$$


Next let us estimate the error $\psi_{2 i}^{n}$. In view of the Taylor expansion $u_{\bar{x}, i}=\frac{\partial u}{\partial x}-\frac{h}{2} \frac{\partial^{2} u}{\partial x^{2}}+$ $O\left(h^{2}\right)$, after some tedious manipulations we have

$$
\psi_{2 i}^{n}=a \sigma \tau \frac{\partial^{2} u}{\partial t \partial x}+a \sigma \frac{h}{2} \frac{\partial^{2} u}{\partial x^{2}}+O\left(\tau^{2}+h^{2}\right) .
$$

It remains to estimate $\psi_{3 i}^{n}$ and $\psi_{4 i}^{n}$. Let us substitute the Taylor expansion

$$
\begin{gathered}
(\varphi(\hat{u}))_{x, i}=\frac{\partial \varphi(\hat{u})}{\partial x}+\frac{h}{2} \frac{\partial^{2} \varphi(\hat{u})}{\partial x^{2}}+\frac{h^{2}}{3 !} \frac{\partial^{3} \varphi(\hat{u})}{\partial x^{3}}+\frac{h^{3}}{4 !}\left(\frac{\partial^{4} \varphi\left(\hat{u}_{\xi_{1 i}}\right)}{\partial x^{4}}\right), \\
(\varphi(\hat{u}))_{\bar{x}, i}=\frac{\partial \varphi(\hat{u})}{\partial x}-\frac{h}{2} \frac{\partial^{2} \varphi(\hat{u})}{\partial x^{2}}+\frac{h^{2}}{3 !} \frac{\partial^{3} \varphi(\hat{u})}{\partial x^{3}}-\frac{h^{3}}{4 !}\left(\frac{\partial^{4} \varphi\left(\hat{u}_{\xi_{2 i}}\right)}{\partial x^{4}}\right), \\
\hat{u}_{\xi_{1 i}}=u\left(\xi_{1 i}, t_{n+1}\right), \xi_{1 i} \in\left(x_{i}, x_{i+1}\right), \hat{u}_{\xi_{2 i}}=u\left(\xi_{2 i}, t_{n+1}\right), \xi_{2 i} \in\left(x_{i-1}, x_{i}\right),
\end{gathered}
$$

to the error $\psi_{3 i}^{n}$

$$
\begin{aligned}
\psi_{3 i}^{n} & =\sigma\left(\frac{\hat{u}_{i+1}-\hat{u}_{i}}{h}-\frac{\partial \hat{u}}{\partial x}\right) \frac{\partial \varphi(\hat{u})}{\partial x}+\sigma\left(\frac{\hat{u}_{i+1}+\hat{u}_{i}}{2}-\hat{u}_{i}\right) \frac{\partial^{2} \varphi(\hat{u})}{\partial x^{2}} \\
& +h^{2} \sigma\left(\frac{1}{3 !} \hat{u}_{x, i} \frac{\partial^{3} \varphi(\hat{u})}{\partial x^{3}}+\frac{\hat{u}_{i+1}}{4} \frac{\partial^{4} \varphi\left(\hat{u}_{\xi_{1 i}}\right)}{\partial x^{4}}+\frac{\hat{u}_{i}}{4} \frac{\partial^{4} \varphi\left(\hat{u}_{\xi_{2 i}}\right)}{\partial x^{4}}\right) .
\end{aligned}
$$

Taking into account the following estimation and transformations

$$
\begin{gathered}
\left|\hat{u}_{x, i}\right|=\left|\frac{1}{h} \int_{x_{i}}^{x_{i+1}} \frac{\partial u\left(\xi, t_{n+1}\right)}{\partial \xi} d \xi\right| \leq \frac{1}{h} \int_{x_{i}}^{x_{i+1}}\left|\frac{\partial u\left(\xi, t_{n+1}\right)}{\partial \xi}\right| d \xi \leq \max _{(x, t) \in \bar{Q}_{T}}\left|\frac{\partial u}{\partial x}(x, t)\right|, \\
\hat{u}_{x, i}=\frac{\partial \hat{u}}{\partial x}+\frac{h}{2} \frac{\partial^{2} \hat{u}}{\partial x^{2}}+O\left(h^{2}\right), \quad \frac{\hat{u}_{i+1}+\hat{u}_{i}}{2}-\hat{u}_{i}=\frac{h}{2} \frac{\partial \hat{u}}{\partial x}+O\left(h^{2}\right),
\end{gathered}
$$

we are now in a position to estimate the error $\psi_{3 i}^{n}$

$$
\psi_{3 i}^{n}=\sigma \frac{h}{2} \frac{\partial^{2} \hat{u}}{\partial x^{2}} \frac{\partial \varphi(\hat{u})}{\partial x}+\sigma \frac{h}{2} \frac{\partial \hat{u}}{\partial x} \frac{\partial^{2} \varphi(\hat{u})}{\partial x^{2}}+O\left(h^{2}\right) .
$$

An argument similar to the one used above shows that

$$
\psi_{4 i}^{n}=-(1-\sigma) \frac{h}{2} \frac{\partial^{2} u}{\partial x^{2}} \frac{\partial \varphi(u)}{\partial x}-(1-\sigma) \frac{h}{2} \frac{\partial u}{\partial x} \frac{\partial^{2} \varphi(u)}{\partial x^{2}}+O\left(h^{2}\right) .
$$

We use the relation (14) to obtain the estimations

$$
\psi_{3 i}^{n}+\psi_{4 i}^{n}=(\sigma-0.5) h\left(\frac{\partial^{2} \bar{u}}{\partial x^{2}} \frac{\partial \varphi(\bar{u})}{\partial x}+\frac{\partial \bar{u}}{\partial x} \frac{\partial^{2} \varphi(\bar{u})}{\partial x^{2}}\right)+O\left(\tau^{2}+h^{2}\right) .
$$

Now, it is straightforward to show that the error of approximation is

$$
\psi_{i}^{n}=O(\tau+h) .
$$

Thus, the difference scheme (24) - (26) has the first order of approximation.

Remark 1. A difference scheme of the second order $O\left(\tau^{2}+h^{2}\right)$ of approximation for $\alpha=\sigma=0.5$ has the form

$$
y_{t, i}+a \alpha y_{x, i}^{n+1}+a(1-\alpha) y_{\bar{x}, i}^{n}=\sigma \Lambda y_{i}^{n+1}+(1-\sigma) \Lambda^{-} y_{i}^{n}, \alpha, \sigma \in[0,1] .
$$

For $\alpha=0$ the above scheme reduces to the scheme (24). Scheme (27) is exact for the travelling wave solutions under the conditions $\gamma=\frac{(a+c) \tau}{h}=1, \alpha=\sigma$. In this case the 
linear approximation is treated as a finite scheme $y_{t}+a \alpha \hat{y}_{x}+a(1-\alpha) y_{\bar{x}}=b y_{\bar{x} x}^{(\sigma)}$, where $a, b$ are the positive constants, which is monotone and stable under two conditions: $a h / b<\sigma / \alpha, \tau \leq h^{2} /(2 b(1-\sigma)+a h(1-\alpha))$. The first condition is connected with the so-called Peclet number $P e=a h / b$ and for a wide class of problems imposes very small grid steps. As a simple example, the convection-diffusion problem with the small parameter, i.e., $b \ll 1$ can be considered.

Another way to increase the order of approximation and avoid the restriction on the cell Peclet number at the same time is constructing a difference scheme with the regularization

$$
y_{t, i}+\frac{q_{i} a}{k\left(y_{i}\right)} y_{\bar{x}, i}=\hat{\kappa}_{i} \sigma \Lambda \hat{y}_{i}+\kappa_{i}(1-\sigma) \Lambda^{-} y_{i}, 0 \leq \sigma \leq 1,
$$

where $\kappa_{i}=\frac{1}{1+R_{i}}, R_{i}=\frac{a h}{2 k\left(y_{i}\right)}, q_{i}=0.5\left(k\left(y_{i-1}\right)+k\left(y_{i}\right)\right)$. The approximation error of the above scheme is $O\left(\tau+h^{2}\right)$ for $\sigma=0.5$. For $k(u) \equiv b$, the scheme (28) reduces to

$$
y_{t, i}+a y_{\bar{x}, i}=\kappa \sigma \Lambda \hat{y}_{i}+\kappa(1-\sigma) \Lambda^{-} y_{i}, 0 \leq \sigma \leq 1,
$$

with $\kappa=\frac{1}{1+R}, \quad R=\frac{a h}{2 b}$. More information about the difference schemes with a regularization can be found in $[\mathbf{1 1}]$.

\subsection{The proof of the main result}

In the present section the exactness of the scheme (24) - (26) will be considered.

First we will need the following lemma.

Lemma 3. For the travelling-wave solution $u(x, t)=U(x-(a+c) t), a, c=$ const, $a+c>0$, of the equation (20) the following equality is fulfilled

$$
\sigma\left(\hat{u}(\varphi(\hat{u}))_{\bar{x}}\right)_{x, i}+(1-\sigma)\left(u(\varphi(u))_{x}\right)_{\bar{x}, i}=-c \sigma \hat{u}_{x, i}-c(1-\sigma) u_{\bar{x}, i} .
$$

Proof. First, let us notice that for the travelling wave solution $u(x, t)=U(x-$ $(a+c) t)$ there follows

$$
\frac{\partial}{\partial x}\left(u \frac{\partial \varphi(u)}{\partial x}\right)=\frac{\partial u}{\partial t}+a \frac{\partial u}{\partial x}=-(a+c) U^{\prime}+a U^{\prime}=-c \frac{\partial u}{\partial x} .
$$

From the above equality we get

$$
\frac{\partial}{\partial x}(\varphi(u))=-c
$$

Integrating the equation (29) for $t=t_{n+1}$ on the closed intervals $\left[x_{i-1}, x_{i}\right]$ and $\left[x_{i}, x_{i+1}\right]$ we have

$$
(\varphi(\hat{u}))_{\bar{x}, i}=-c,(\varphi(\hat{u}))_{\bar{x}, i+1}=-c .
$$

After some tedious manipulation we obtain

$$
\left(\hat{u}(\varphi(\hat{u}))_{\bar{x}}\right)_{x, i}=-c \hat{u}_{x, i} .
$$


In a similar way we get

$$
\left(u(\varphi(u))_{x}\right)_{\bar{x}, i}=-c u_{\bar{x}, i} .
$$

Now it is straightforward to show that

$$
\sigma\left(\hat{u}(\varphi(\hat{u}))_{\bar{x}}\right)_{x, i}+(1-\sigma)\left(u(\varphi(u))_{x}\right)_{\bar{x}, i}=-c \sigma \hat{u}_{x, i}-c(1-\sigma) u_{\bar{x}, i} .
$$

The essential observation is that the above Lemma gives the condition on equivalence of the convection-diffusion equation and the transport equation. In the next theorem we give the condition under which the scheme (24) - (26) is exact.

Theorem 2. If $\gamma=\frac{(a+c) \tau}{h}=1$, then the difference scheme $(24)$ - (26) is exact for the travelling wave solution of the form $u(x, t)=U(x-(a+c) t), a+c>0$.

Proof. From Lemma 3 the following equality follows

$$
\psi_{i}^{n}=-u_{t, i}-c \sigma \hat{u}_{x, i}-(a+c(1-\sigma)) u_{\bar{x}, i} .
$$

For $\gamma=1$ we have

$$
u_{i+1}^{n+1}=U\left(x_{i+1}-(a+c) t_{n+1}\right)=U\left(x_{i}-(a+c) t_{n}\right)=u_{i}^{n},
$$

and the approximation error satisfies the relationships

$$
\begin{gathered}
\psi_{i}^{n}=\left(-\frac{c \sigma \tau}{h} u_{i+1}^{n+1}+\frac{c \sigma \tau}{h} u_{i}^{n+1}-\frac{c(1-\sigma) \tau}{h} u_{i}^{n}+\frac{c(1-\sigma) \tau}{h} u_{i}^{n}-\frac{a \tau}{h} u_{i}^{n}-\frac{a \tau}{h} u_{i-1}^{n}\right) / \tau+ \\
+\left(-u_{i}^{n+1}+u_{i}^{n}\right) / \tau=\left((c \tau / h-1) u_{i}^{n+1}+(1-c \tau / h) u_{i}^{n}-a \tau / h u_{i}^{n}-a \tau / h u_{i-1}^{n}\right) / \tau \\
=\left((\gamma-1) u_{i-1}^{n}+(1-\gamma) u_{i}^{n}\right) / \tau=0 .
\end{gathered}
$$

Hence, the difference scheme (24) - (26) is exact.

Remark 2. For the travelling wave solutions $u(x, t)=U(x-(a+c) t)$ under the condition $\sigma=0.5(a+c) / c$ the difference scheme $(24)$ - (26) is equivalent to the scheme (10) and its approximation error is

$$
\psi_{i}^{n}=-u_{t, i}-0.5(a+c) \hat{u}_{x, i}-0.5(a+c) u_{\bar{x}, i} .
$$

Thus it has tthe second order of approximation for $\gamma \neq 1$.

Remark 3. For an arbitrary sign of the coefficient $a$ the following difference scheme

$$
y_{t}+\frac{a+|a|}{2} y_{\bar{x}}^{n}+\frac{a-|a|}{2} y_{x}^{n}=\sigma \Lambda \hat{y}+(1-\sigma) \Lambda^{-} y
$$

is still exact for the travelling wave solutions $u(x, t)=U(x-(a+c) t)$ with $\gamma=$ $(c+a) \tau / h, c+a>0$.

Remark 4. The scheme (24) - (26) is nonlinear even in the linear case and the following iterative method is used for its implementation

$$
\frac{{\stackrel{s+1}{y}-y_{i}^{n}}_{\tau}}{\tau}+a y_{\bar{x}, i}^{n}=\sigma\left[\stackrel{s}{y}\left(\varphi(\stackrel{s}{y})+\varphi^{\prime}(\stackrel{s}{y})\left({ }^{s+1} y^{-1} y\right)\right)_{\bar{x}}\right]_{x, i}+(1-\sigma) \Lambda^{-} y_{i}^{n} .
$$


Pobrane z czasopisma Annales AI- Informatica http://ai.annales.umcs.pl

Data: 26/04/2023 14:49:40

Exact difference schemes and schemes of higher...

The iteration process is terminated, when $\|\stackrel{s+1}{y}-\stackrel{s}{y}\|_{C} \leq \epsilon$ for some $s=S$. Then we advance to the next level with $y_{i}^{n+1}={ }^{S+1}{ }_{i}, i=1, \ldots, N-1$. The initial approximation is taken from the explicit scheme $(24)(\sigma=0)$.

\subsection{Numerical experiment for a linear equation}

In this section we carry out numerical experiments to illustrate the convergence of the exact difference scheme for a linear convection-diffusion equation.

Consider the equation (20) with $k(u)=b$ and $b=$ const $>0$ together with the input conditions

$$
u(x, 0)=e^{-0.5 x}, u(0, t)=e^{(0.5 a+0.25 b) t}, u(l, t)=e^{(0.5 a+0.25 b) t-0.5 x} .
$$

The solution of this problem is $u(x, t)=e^{0.5((a+0.5 b) t-x)}$. In this case $c=0.5 b$ and the considered scheme is exact for $(a+0.5 b) \tau / h=1$. Moreover, for $\sigma=(2 a+b) /(2 b)$ and $(a+0.5 b) \tau / h \neq 1$ the scheme has the second order of approximation.

Table 3. $\sigma=0.5, \frac{(a+0.5 b) \tau}{h}=1, a=b=1, l=T=1, \epsilon=1.0 \cdot 10^{-15}$.

\begin{tabular}{|l|l|c|c|}
\hline$h$ & $\tau$ & $\max _{t_{n} \in \bar{\omega}_{\tau}}\left\|y^{n}-u^{n}\right\|_{\bar{C}}$ & number of iterations \\
\hline 0.5 & $0.3(3)$ & $1.08 \cdot 10^{-19}$ & 1 \\
\hline 0.05 & $0.03(3)$ & $3.79 \cdot 10^{-17}$ & 2 \\
\hline 0.005 & $0.003(3)$ & $1.55 \cdot 10^{-17}$ & 2 \\
\hline 0.0005 & $0.0003(3)$ & $1.35 \cdot 10^{-16}$ & 2 \\
\hline
\end{tabular}

Table 4. $\sigma=0.5, \frac{(a+0.5 b) \tau}{h} \neq 1, a=b=1, l=T=1, \epsilon=1.0 \cdot 10^{-10}$.

\begin{tabular}{|l|l|c|c|}
\hline$h$ & $\tau$ & $\max _{t_{n} \in \bar{\omega}_{\tau}}\left\|y^{n}-u^{n}\right\|_{\bar{C}}$ & number of iterations \\
\hline 0.1 & 0.1 & $1.15 \cdot 10^{-03}$ & 6 \\
\hline 0.01 & 0.01 & $1.19 \cdot 10^{-04}$ & 4 \\
\hline 0.001 & 0.001 & $1.19 \cdot 10^{-05}$ & 3 \\
\hline 0.0005 & 0.0005 & $5.96 \cdot 10^{-06}$ & 2 \\
\hline
\end{tabular}

Table 5. $\sigma=(2 a+b) /(2 b), \frac{(a+0.5 b) \tau}{h} \neq 1, a=b=1, l=T=1, \epsilon=1.0 \cdot 10^{-10}$.

\begin{tabular}{|l|l|c|c|}
\hline$h$ & $\tau$ & $\max _{t_{n} \in \bar{\omega}_{\tau}}\left\|y^{n}-u^{n}\right\|_{\bar{C}}$ & number of iterations \\
\hline 0.1 & 0.1 & $7.27 \cdot 10^{-06}$ & 7 \\
\hline 0.01 & 0.01 & $7.43 \cdot 10^{-08}$ & 4 \\
\hline 0.001 & 0.001 & $7.46 \cdot 10^{-10}$ & 2 \\
\hline 0.0005 & 0.0005 & $1.86 \cdot 10^{-10}$ & 2 \\
\hline
\end{tabular}

The number of iterations needed in the nonlinear scheme is sufficiently small. 


\subsection{Numerical experiment for a nonlinear equation}

In this section we carry out numerical experiments to illustrate the convergence of the exact difference scheme for a nonlinear convection-diffusion equation.

Consider the equation (20) with $k(u)=\chi_{0} u^{\beta}$ and $\chi_{0}=$ const $>0, \beta=$ const $>0$ together with the input conditions

$$
\begin{gathered}
u(x, 0)=0, u(0, t)=b(D+a) t^{1 / \beta} D^{-1 / \beta}, \\
u(l, t)=\left\{\begin{array}{ll}
\frac{b}{D^{1 / \beta}}((D+a) t-l)^{1 / \beta}, & l \leq(D+a) t, \\
0, & (D+a) t<l,
\end{array}, b=\text { const }>0, D=\sqrt{\chi_{0} b^{\beta} / \beta}\right.
\end{gathered}
$$

The solution of this problem is

$$
u(x, t)=\left\{\begin{array}{ll}
\frac{b}{D^{1 / \beta}}((D+a) t-x)^{1 / \beta}, & 0 \leq x \leq(D+a) t, \\
0, & x>(D+a) t
\end{array} .\right.
$$

In this case $c=D$ and the considered scheme is exact for $(a+D) \tau / h=1$.

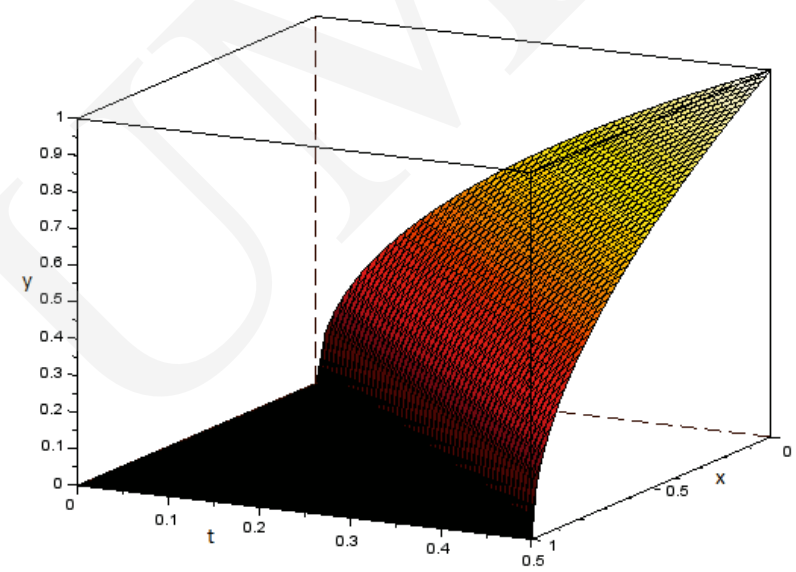

Fig. 2. The approximate solution for $\sigma=0.5, \tau=0.01, h=0.02$

Table 6. $\sigma=0.5, \frac{(a+D) \tau}{h}=1, a=b=1, \chi_{0}=\beta=2, l=1, T=0.5$, $\epsilon=1.0 \cdot 10^{-16}$.

\begin{tabular}{|l|l|c|c|}
\hline$h$ & $\tau$ & $\max _{t_{n} \in \bar{\omega}_{\tau}}\left\|y^{n}-u^{n}\right\|_{\bar{C}}$ & number of iterations \\
\hline 0.1 & 0.05 & $9.76 \cdot 10^{-19}$ & 1 \\
\hline 0.01 & 0.005 & $4.39 \cdot 10^{-18}$ & 2 \\
\hline
\end{tabular}




\section{Future research}

In the future our research will focus on the two-dimensional case of the convectiondiffusion equation. The travelling wave solution will be still considered and the equivalence to the transport equation will be crucial.

In the domain $\bar{Q}_{T}=\bar{\Omega} \times[0, T]$ let us consider the initial-boundary value problem for a transport equation with the positive coefficients $a_{1}, a_{2}>0$

$$
\begin{gathered}
\frac{\partial u}{\partial t}+a_{1} \frac{\partial u}{\partial x_{1}}+a_{2} \frac{\partial u}{\partial x_{2}}=0,(\mathbf{x}, t) \in \Omega \times(0, T], \\
u(\mathbf{x}, 0)=u_{0}(\mathbf{x}), \quad \mathbf{x} \in \bar{\Omega},\left.\quad u\right|_{\mathbf{x} \in \partial \Omega}=g(\mathbf{x}, t),(\mathbf{x}, t) \in \partial \Omega \times(0, T],
\end{gathered}
$$

where

$\partial \Omega=\left\{x \in \bar{\Omega}: x_{k}=0, k=1,2\right\} \bar{\Omega}=\left\{\mathbf{x}=\left(x_{1}, x_{2}\right): 0 \leq x_{k} \leq l_{k}, k=1,2\right\}=\Omega \cup \partial \Omega$.

Let us introduce the uniform grids $\bar{\omega}_{h_{k}}=\left\{x_{k, i}: x_{k, i}=i h_{k}, i=0, \ldots, N_{k}, h_{k} N_{k}=l_{k}\right\}$, $k=1,2, \bar{\omega}_{h}=\bar{\omega}_{h_{1}} \times \bar{\omega}_{h_{2}}, \omega_{h}=\bar{\omega}_{h} \cap \Omega$ and $\partial \omega_{h}=\bar{\omega}_{h} \cap \partial \Omega$.

The following difference scheme approximating the problem (31) - (32) will be under consideration $[\mathbf{1 6}, \mathbf{4}]$

$$
\begin{gathered}
y_{t}+a_{1} y_{\left(\mu_{2}\right) \bar{x}_{1}}+a_{2} y_{\left(\mu_{1}\right) \bar{x}_{2}}=0, \mathbf{x} \in \omega_{h}, t \in \omega_{\tau}, \\
y(\mathbf{x}, 0)=u_{0}(\mathbf{x}), \mathbf{x} \in \bar{\omega}_{h},\left.\quad y\right|_{\mathbf{x} \in \partial \Omega}=g(\mathbf{x}, t), \quad(\mathbf{x}, t) \in \partial \omega_{h} \times \omega_{\tau} .
\end{gathered}
$$

Here $y_{\left(\mu_{1}\right)}=\mu_{1} y_{i_{1}, i_{2}}+\left(1-\mu_{1}\right) y_{i_{1}-1, i_{2}}$ and $y_{\left(\mu_{2}\right)}=\mu_{2} y_{i_{1}, i_{2}}+\left(1-\mu_{2}\right) y_{i_{1}, i_{2}-1}$ for $0 \leq \mu_{1}, \mu_{2} \leq 1$. The scheme (33) - (34) is exact under conditions

$$
\mu_{1}+\mu_{2}=1, \gamma_{1}=\gamma_{2}=1, \gamma_{k}=a_{k} \tau / h, k=1,2 .
$$

It is monotone and stable under the condition

$$
0 \leq \mu_{1}, \mu_{2} \leq 1, \max \left\{\gamma_{1}, \gamma_{2}\right\} \leq \gamma_{1} \mu_{2}+\gamma_{2} \mu_{1} \leq 1
$$

\section{References}

[1] Samarskii A. A., Matus P. P., Vabishchevich P. N., Difference Schemes with Operator Factors, Kluwer Academic Publishers, Netherlands (2002).

[2] Gavrilyuk I., Hermann M., Makarov V., Kutniv M. V., Exact and Truncated Difference Schemes for Boundary Value ODEs, Springer, Berlin (2011).

[3] Micken R. E., Applications of Nonstandard Finite Difference Schemes, World Scientific Publishing, Singapore (2000).

[4] Mickens R. E., Exact finite difference schemes for two-dimensional advection equations, Journal of Sound and Vibration 207(3) (1997): 426.

[5] Paradzinska A., Matus P., High Accuracy Difference Schemes for Nonlinear Transfer Equation $\frac{\partial u}{\partial t}+u \frac{\partial u}{\partial x}=f(u)$, Mathematical Modelling and Analysis 12(4) (2007): 469.

[6] Rucker S., Exact Finite Difference Scheme for an Advection-Reaction Equation, Journal of Difference Equations and Applications 9(11) (2003): 1007.

[7] Matus P., Irkhin U., Lapinska-Chrzczonowicz M., Exact difference schemes for time-dependent problems, Computational Methods In Applied Mathematics 5(4) (2005): 422. 
[8] Matus P., Irkhin U., Lapinska-Chrzczonowicz M., Lemeshevsky S. V., On exact finite-difference schemes for hyperbolic and elliptic equations. Differentsial'nye, Uravneniya 43(7) (2007): 978.

[9] Gilding B. H., Kersner R., Travelling Waves in Nonlinear Diffusion-Convection Reaction, Birkhauser Verlag, Berlin (2004).

[10] Volpert A. I., Volpert V. A., Volpert V. A., Traveling Wave Solutions of Parabolic Systems, American Mathematical Society, Rhode Island (2000).

[11] Samarskii A. A., The theory of difference schemes, Marcel Dekker Inc., New York - Basel (2001).

[12] Smarskii A. A., Gulin A. W., Numerical methods, Nauka, Moscow (1989); (in Russian).

[13] Matus P. P., Kirshtein A. A., Irkhin U. A., Exact difference schemes for the system of acoustic equations and analysis of Riemann problem, J. Numer. Appl. Math. 105(2) (2011): 83.

[14] Lapinska-Chrzczonowicz M., Difference schemes of arbitrary order of accuracy for semilinear parabolic equations, Annales UMCS, Informatica 10(2) (2010): 93.

[15] Hundsdorfer W., Verwer J. G., Numerical Solution of Time-Dependent Advection-DiffusionReaction Equations, Springer, Berlin (2003).

[16] Lemeshevsky S. V., Matus P. P., Yakubuk R. M., Two-layered higher-order difference schemes for the convection-diffusion equation, Doklady of The National Academy of Sciences of Belarus 56(2) 2012: 15. 